\title{
The Neutrophil Percentage-to-Albumin Ratio Is Associated with All-Cause Mortality in Critically Ill Patients with Acute Kidney Injury
}

\author{
Benji Wang $\mathbb{D}$, Diwen Li, Bihuan Cheng $\mathbb{D}$, Binyu Ying, and Yuqiang Gong \\ Department of Anesthesiology, Critical Care and Pain Medicine, \\ The Second Affiliated Hospital and Yuying Children's Hospital of Wenzhou Medical University, Wenzhou 325000, \\ Zhejiang, China \\ Correspondence should be addressed to Yuqiang Gong; gongyuqiang@wmu.edu.cn
}

Received 7 November 2019; Revised 29 January 2020; Accepted 30 January 2020; Published 18 February 2020

Academic Editor: Valeria Cavalcanti Rolla

Copyright (c) 2020 Benji Wang et al. This is an open access article distributed under the Creative Commons Attribution License, which permits unrestricted use, distribution, and reproduction in any medium, provided the original work is properly cited.

\begin{abstract}
Background. There is no evidence to suggest the predictive power of neutrophil percentage-to-albumin ratio (NPAR) in patients with acute kidney injury (AKI). We hypothesized that NPAR would correlate with all-cause mortality in critically ill patients with AKI. Methods. From the MIMIC-III V1.4 database, we extracted demographics, vital signs, comorbidities, laboratory tests, and other clinical data. The clinical endpoints were 30-, 90- and 365-day all-cause mortality in critically ill patients with AKI. Cox proportional hazards models were used to evaluate the prognostic values of NPAR, and subgroup analyses were performed to measure mortality across various subgroups. Results. A total of 7,481 eligible subjects were enrolled. In multivariate analysis, after adjustments for age, ethnicity, gender, and other confounding factors, higher NPARs were associated with an increased risk of 30-, 90- and 365-day all-cause mortality in critically ill patients with AKI (tertile 3 versus tertile 1: adjusted HR, 95\% CI: 1.48, 1.30-1.69; $1.47,1.31-1.66 ; 1.46,1.32-1.62$, respectively; $P$ trend $<0.01$ ). A similar trend was observed in the NPAR group division by quintiles. Subgroup analysis revealed no significant interactions in most strata. Conclusions. Increased NPAR correlates with increased risk of all-cause mortality in critically ill patients with AKI.
\end{abstract}

\section{Introduction}

Acute kidney injury (AKI) is defined as a sudden deterioration of renal function and is associated with high morbidity and mortality [1], especially for critically ill patients. In the US, $6-24 \%$ of critically ill patients in intensive care units (ICUs) have AKI [2] and the mortality rate of these patients is as high as $60-70 \%$ [3], greatly increasing healthcare costs and imposing substantial healthcare burdens. Given the poor prognosis of AKI in critical illness, finding novel biomarkers to identify the severity of AKI and adopting early effective interventions to improve survival are critical. Investigators have sought several few biomarkers of mortality in AKI [4, 5]; nevertheless, these efforts have been largely unsuccessful.

The pathogenesis of AKI has not been fully elucidated; progression of AKI may be associated with systemic inflammatory [6-8]. Leukocytes, including neutrophils, produce inflammatory mediators such as cytokines and chemokines that damage the kidneys. Albumin is a crucial protein with several functions, including osmotic pressure regulation and antioxidant and anti-inflammatory effects $[9,10]$; it too has been associated with AKI [11]. Therefore, we hypothesize that neutrophil percentage-to-albumin (NPAR) could serve as an inflammation-based prognostic score. Neutrophil-to-albumin ratio (NAR) has been identified as a biomarker predicting prognosis in patients with rectal cancer and end-stage pancreatic cancer [12, 13]. Based on these findings, we have reason to speculate that NPAR may affect the prognosis of AKI in critical illness. To our knowledge, there has been no epidemiological study exploring the association between NPAR and mortality in critically ill patients with AKI. 


\section{Methods}

2.1. Data Source. Similar to our previous studies, we followed the methods of Wang et al. [14, 15]. The Multiparameter Intelligent Monitoring in Intensive Care III version 1.4 (MIMIC-III v1.4) is an openly available dataset. It includes deidentified health data derived from $\sim 40,000$ critical care patients from 2001 to 2012 [16]. To apply for access to the database, we passed the Protecting Human Research Participants exam and obtained a certificate (No. 6182750). The project is approved by the institutional review boards of the Massachusetts Institute of Technology and Beth Israel Deaconess Medical Center and was granted a waiver of informed consent.

2.2. Population Selection Criteria. We restricted the search to adult patients ( $\geq 18$ years) with AKI. The occurrence of AKI was determined on the basis of Kidney Disease: Improving Global Outcomes (KDIGO) definition [17], and Structured Query Language (SQL) for extracting AKI was included in supplementary material. For inclusion, patients needed to be hospitalized in the ICU at first admission for more than two days. Patients who met the following criteria were excluded: (1) no neutrophil percentage and albumin measured during ICU stay and (2) more than $5 \%$ of individual data missing.

2.3. Data Extraction. SQL with PostgreSQL tools (version 9.6) was used to extract the data from MIMIC-III. Demographics, vital signs, comorbidities, laboratory tests, and others were extracted. The comorbidities included coronary artery disease (CAD), congestive heart failure (CHF), atrial fibrillation (AFIB), stroke, renal disease, liver disease, pneumonia, malignancy, and respiratory failure. Laboratory tests included neutrophil percentage, albumin, bicarbonate, creatinine, chloride, glucose, hematocrit, hemoglobin, platelet, sodium, potassium, blood urea nitrogen (BUN), white blood cell (WBC), prothrombin time (PT), activated partial thromboplastin time (APTT), and international normalized ratio (INR). Sequential organ failure assessment (SOFA) score [18] and simplified acute physiology score II (SAPSII) [19] were calculated for each patient at the time of ICU admission. The other extracted data included age, gender, ethnicity, systolic blood pressure (SBP), diastolic blood pressure (DBP), mean blood pressure (MBP), heart rate, respiratory rate, temperature, $\mathrm{SPO} 2, \mathrm{AKI}$ stage, renal replacement therapy, vasopressor use, and length of stay in the ICU. Records containing laboratory tests were extracted within 24 hours after admission to the ICU. Survival information regarding vital status was obtained from the Social Security Death Index records. The endpoints for this analysis were 30-day, 90-day, and 365-day all-cause mortality.

2.4. Statistical Analysis. Baseline characteristics of all patients were stratified by NPAR tertiles. Continuous variables were presented as mean \pm standard deviation (SD), and categorical data were summarized as number or percentage.

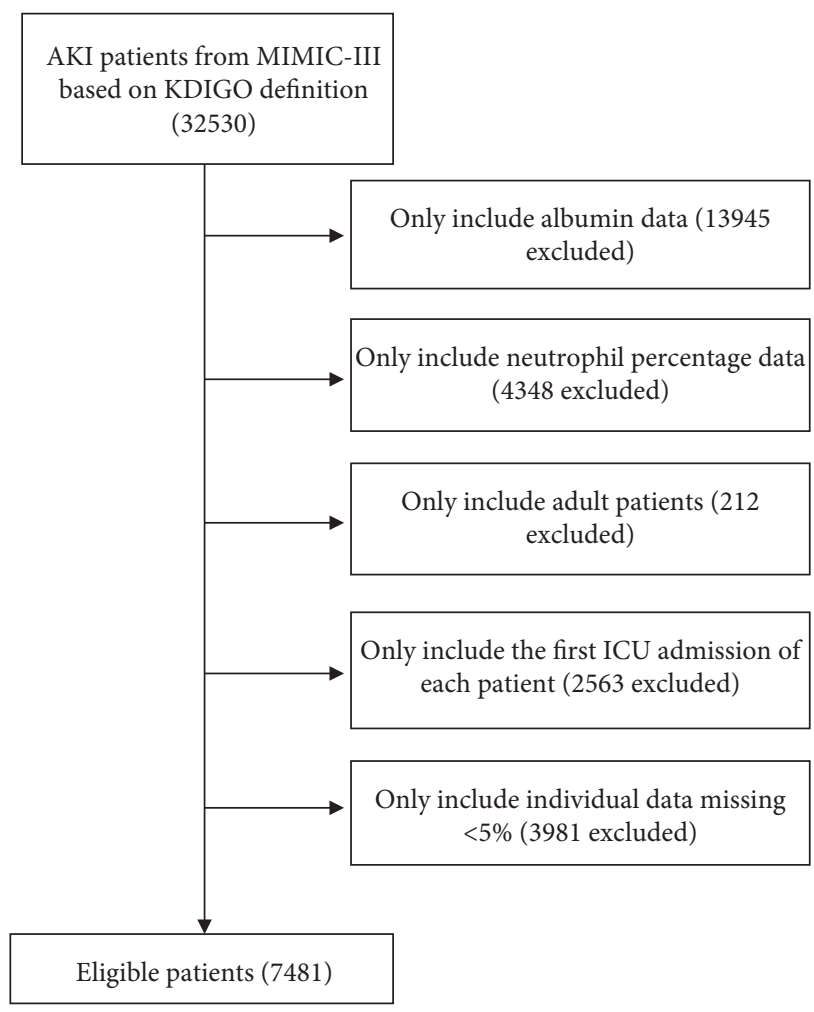

FIGURE 1: Illustration of exclusion and inclusion criteria as utilized to select the final 7481 patients.

We used chi-square or one-way ANOVA to test for differences in categorical or continuous factors among various categories of NPAR. The prognostic values of NPAR were evaluated using Cox proportional hazards models, and the results were presented as hazard ratios (HRs) with $95 \%$ confidence intervals (CIs).

Two multivariate models were constructed on the basis of NPAR group inclusion according to tertiles and quintiles based on 30-, 90-, and 365-day all-cause mortality. The first tertile or quintile was treated as the reference group. In model I, covariates were only adjusted for age, ethnicity, and gender. In model II, we further adjusted for age, ethnicity, gender, AKI stage, CHF, AFIB, liver disease, CAD, stroke, malignancy, respiratory failure, pneumonia, sodium, potassium, chloride, BUN, INR, APTT, platelet, WBC, hematocrit, creatinine, glucose, bicarbonate, vasopressor use, heart rate, SBP, DBP, respiration rate, temperature, SPO2, SOFA, SAPSII, and renal replacement therapy. We selected these confounders based on a change in effect estimate of more than $10 \%$ [20]. Subgroup analyses were performed to evaluate whether the effect of the 30-day mortality differed across various subgroups classified by CHF, AFIB, CAD, stroke, malignancy, liver disease, respiratory failure, pneumonia, AKI stage, WBC, sodium, BUN, INR, potassium, APTT, platelet, hematocrit, creatinine, bicarbonate, glucose, chloride, SBP, DBP, heart rate, respiratory rate, temperature, SPO2, SOFA score, SAPSII score, vasopressor use, and renal replacement therapy.

Receiver-operating characteristic (ROC) curve was performed to measure the sensitivity and specificity of NPAR, 
TABLE 1: Characteristics of the study patients according to neutrophil percentage-to-albumin ratios.

\begin{tabular}{|c|c|c|c|c|}
\hline \multirow{2}{*}{ Characteristics } & \multicolumn{3}{|c|}{ Neutrophil percentage-to-albumin ratios } & \multirow{2}{*}{$P$ value } \\
\hline & $<22.1(n=2492)$ & $\geq 22.1,<28.1 \quad(n=2494)$ & $\geq 28.1 \quad(n=2495)$ & \\
\hline Age (years) & $62.0 \pm 18.1$ & $65.7 \pm 17.0$ & $65.8 \pm 16.6$ & $<0.01$ \\
\hline Gender, $n(\%)$ & & & & $<0.01$ \\
\hline Female & $989(39.7)$ & $1072(43.0)$ & $1184(47.5)$ & \\
\hline Male & $1503(60.3)$ & $1422(57.0)$ & $1311(52.5)$ & \\
\hline Ethnicity, $n(\%)$ & & & & $<0.01$ \\
\hline White & $1706(69.6)$ & $1777(72.4)$ & $1741(71.1)$ & \\
\hline Black & $331(13.5)$ & $231(9.4)$ & $221(9.0)$ & \\
\hline Other & $414(16.9)$ & $448(18.2)$ & $486(19.9)$ & \\
\hline NPAR & $17.4 \pm 4.7$ & $25.0 \pm 1.7$ & $35.4 \pm 7.3$ & $<0.01$ \\
\hline SBP (mmHg) & $119.8 \pm 18.2$ & $118.7 \pm 17.7$ & $114.0 \pm 16.4$ & $<0.01$ \\
\hline $\mathrm{DBP}(\mathrm{mmHg})$ & $62.9 \pm 11.9$ & $60.6 \pm 11.1$ & $58.2 \pm 10.7$ & $<0.01$ \\
\hline MBP (mmHg) & $79.6 \pm 12.1$ & $77.7 \pm 11.4$ & $75.0 \pm 11.1$ & 0.03 \\
\hline Heart rate (beats/minute) & $86.9 \pm 17.9$ & $87.4 \pm 16.9$ & $91.1 \pm 17.1$ & $<0.01$ \\
\hline Respiratory rate (beats/minute) & $19.4 \pm 4.3$ & $19.8 \pm 4.2$ & $20.4 \pm 4.6$ & $<0.01$ \\
\hline Temperature $\left({ }^{\circ} \mathrm{C}\right)$ & $36.9 \pm 0.7$ & $36.8 \pm 0.7$ & $36.8 \pm 0.8$ & $<0.01$ \\
\hline SPO2 (\%) & $97.0 \pm 2.8$ & $97.0 \pm 2.3$ & $97.0 \pm 3.0$ & 0.18 \\
\hline \multicolumn{5}{|l|}{ Comorbidities, $n(\%)$} \\
\hline Coronary artery disease & $360(14.4)$ & $524(21.0)$ & $384(15.4)$ & $<0.01$ \\
\hline Congestive heart failure & $570(22.9)$ & $611(24.5)$ & $447(17.9)$ & $<0.01$ \\
\hline Atrial fibrillation & $543(21.8)$ & $732(29.4)$ & $701(28.1)$ & $<0.01$ \\
\hline Stroke & $257(10.3)$ & $216(8.7)$ & $152(6.1)$ & $<0.01$ \\
\hline Renal disease & $384(15.4)$ & $458(18.4)$ & $431(17.3)$ & 0.02 \\
\hline Liver disease & $203(8.1)$ & $259(10.4)$ & $339(13.6)$ & $<0.01$ \\
\hline Pneumonia & $701(28.1)$ & $827(33.2)$ & $922(37.0)$ & 0.14 \\
\hline Malignancy & $425(17.1)$ & $388(15.6)$ & $595(23.8)$ & $<0.01$ \\
\hline Respiratory failure & $875(35.1)$ & $1015(40.7)$ & $1289(51.7)$ & $<0.01$ \\
\hline \multicolumn{5}{|l|}{ Laboratory parameters } \\
\hline Neutrophil (\%) & $65.8 \pm 20.3$ & $82.1 \pm 8.8$ & $85.2 \pm 8.0$ & $<0.01$ \\
\hline Albumin (g/dl) & $3.8 \pm 0.6$ & $3.3 \pm 0.4$ & $2.5 \pm 0.4$ & $<0.01$ \\
\hline Bicarbonate $(\mathrm{mmol} / \mathrm{L})$ & $20.7 \pm 5.5$ & $20.7 \pm 5.5$ & $19.6 \pm 5.7$ & $<0.01$ \\
\hline Creatinine $(\mathrm{mEq} / \mathrm{L})$ & $1.5 \pm 1.7$ & $1.7 \pm 1.7$ & $1.8 \pm 1.7$ & $<0.01$ \\
\hline Chloride $(\mathrm{mmol} / \mathrm{L})$ & $100.6 \pm 6.8$ & $101.0 \pm 7.1$ & $102.5 \pm 7.7$ & $<0.01$ \\
\hline Glucose $(\mathrm{mg} / \mathrm{dl})$ & $143.1 \pm 50.0$ & $147.1 \pm 48.5$ & $146.0 \pm 52.4$ & $<0.01$ \\
\hline Hematocrit (\%) & $31.2 \pm 6.5$ & $30.1 \pm 6.2$ & $27.7 \pm 5.6$ & $<0.01$ \\
\hline Hemoglobin (g/dl) & $10.7 \pm 2.3$ & $10.2 \pm 2.1$ & $9.3 \pm 1.9$ & $<0.01$ \\
\hline Platelet $(109 / \mathrm{L})$ & $183.0 \pm 99.5$ & $201.1 \pm 111.3$ & $203.4 \pm 139.0$ & $<0.01$ \\
\hline Sodium $(\mathrm{mmol} / \mathrm{L})$ & $136.3 \pm 5.4$ & $136.0 \pm 5.8$ & $135.9 \pm 6.2$ & 0.01 \\
\hline Potassium $(\mathrm{mmol} / \mathrm{L})$ & $3.7 \pm 0.6$ & $3.8 \pm 0.6$ & $3.7 \pm 0.7$ & $<0.01$ \\
\hline BUN (mg/dl) & $26.0 \pm 21.7$ & $32.7 \pm 25.1$ & $35.9 \pm 26.5$ & $<0.01$ \\
\hline WBC $(109 / \mathrm{L})$ & $9.7 \pm 12.8$ & $11.1 \pm 6.8$ & $12.8 \pm 7.6$ & $<0.01$ \\
\hline PT (seconds) & $14.8 \pm 4.9$ & $15.6 \pm 6.1$ & $16.0 \pm 5.1$ & $<0.01$ \\
\hline APTT (seconds) & $30.6 \pm 11.8$ & $31.5 \pm 12.1$ & $33.9 \pm 12.7$ & $<0.01$ \\
\hline INR & $1.4 \pm 0.8$ & $1.4 \pm 0.7$ & $1.5 \pm 0.7$ & $<0.001$ \\
\hline \multicolumn{5}{|l|}{ Scoring systems } \\
\hline SOFA & $5.1 \pm 3.6$ & $5.5 \pm 3.4$ & $6.6 \pm 3.8$ & $<0.01$ \\
\hline SAPSII & $37.9 \pm 15.5$ & $40.7 \pm 13.9$ & $45.8 \pm 15.4$ & $<0.01$ \\
\hline AKI stage, $n(\%)$ & & & & $<0.01$ \\
\hline Stage 1 & $641(25.7)$ & $557(22.3)$ & $484(19.4)$ & \\
\hline Stage 2 & $386(15.5)$ & $413(16.6)$ & $436(17.5)$ & \\
\hline Stage 3 & $1465(58.8)$ & $1524(61.1)$ & $1575(63.1)$ & \\
\hline Renal replacement therapy, $n(\%)$ & $214(8.6)$ & $253(10.1)$ & $322(12.9)$ & $<0.01$ \\
\hline Vasopressor use, $n(\%)$ & $841(33.7)$ & $921(36.9)$ & $1247(50.0)$ & $<0.01$ \\
\hline ICU LOS (days) & $4.9 \pm 6.4$ & $5.7 \pm 7.2$ & $7.1 \pm 8.7$ & $<0.01$ \\
\hline 30-day mortality, $n(\%)$ & $386(15.5)$ & $510(20.4)$ & $797(31.9)$ & $<0.01$ \\
\hline 90-day mortality, $n(\%)$ & $522(20.9)$ & $688(27.6)$ & $1032(41.4)$ & $<0.01$ \\
\hline 365 -day mortality, $n(\%)$ & $702(28.2)$ & $939(37.7)$ & $1283(51.4)$ & $<0.01$ \\
\hline
\end{tabular}

NPAR: neutrophil percentage-to-albumin ratio; SBP: systolic blood pressure; DBP: diastolic blood pressure; MBP: mean blood pressure; WBC: white blood cell; BUN: blood urea nitrogen; PT: prothrombin time; APTT: activated partial thromboplastin time; INR: international normalized ratio; SOFA: sequential organ failure assessment; SAPSII: simplified acute physiology score II; AKI: acute kidney injury; ICU: intensive care unit; LOS: length of stay. 
TABLE 2: HRs (95\% CIs) for all-cause mortality across groups of neutrophil percentage-to-albumin ratios.

\begin{tabular}{|c|c|c|c|c|c|c|}
\hline \multirow{2}{*}{ NAR } & \multicolumn{2}{|c|}{ Nonadjusted } & \multicolumn{2}{|c|}{ Model I } & \multicolumn{2}{|c|}{ Model II } \\
\hline & HR (95\% CIs) & $P$ value & HR (95\% CIs) & $P$ value & HR (95\% CIs) & $P$ value \\
\hline \multicolumn{7}{|c|}{ 30-day all-cause mortality } \\
\hline \multicolumn{7}{|c|}{ Tertiles } \\
\hline$<22.1$ & $1.0(\mathrm{ref})$ & & 1.0 (ref) & & 1.0 (ref) & \\
\hline$\geq 22.1,<28.1$ & $1.35(1.18,1.54)$ & $<0.01$ & $1.27(1.11,1.45)$ & $<0.01$ & $1.20(1.05,1.38)$ & 0.01 \\
\hline$\geq 28.1$ & $2.24(1.99,2.53)$ & $<0.01$ & $2.11(1.86,2.39)$ & $<0.01$ & $1.48(1.30,1.69)$ & $<0.01$ \\
\hline$P$ trend & $<0.01$ & & $<0.01$ & & $<0.01$ & \\
\hline \multicolumn{7}{|l|}{ Quintiles } \\
\hline$<19.6$ & 1.0 (ref) & & 1.0 (ref) & & 1.0 (ref) & \\
\hline$\geq 19.6,<23.2$ & $1.04(0.87,1.24)$ & 0.67 & $0.95(0.79,1.14)$ & 0.58 & $0.97(0.80,1.17)$ & 0.74 \\
\hline$\geq 23.2,<26.7$ & $1.29(1.09,1.53)$ & $<0.01$ & $1.18(0.99,1.40)$ & 0.06 & $1.13(0.94,1.35)$ & 0.19 \\
\hline$\geq 26.7,<31.8$ & $1.76(1.49,2.07)$ & $<0.01$ & $1.56(1.32,1.84)$ & $<0.01$ & $1.33(1.12,1.58)$ & $<0.01$ \\
\hline$\geq 31.8$ & $2.48(2.13,2.90)$ & $<0.01$ & $2.29(1.96,2.68)$ & $<0.01$ & $1.48(1.25,1.75)$ & $<0.01$ \\
\hline$P$ trend & $<0.01$ & & $<0.01$ & & $<0.01$ & \\
\hline \multicolumn{7}{|c|}{ 90-day all-cause mortality } \\
\hline \multicolumn{7}{|c|}{ Tertiles } \\
\hline$<22.1$ & 1.0 (ref) & & 1.0 (ref) & & 1.0 (ref) & \\
\hline$\geq 22.1,<28.1$ & $1.36(1.22,1.53)$ & $<0.01$ & $1.28(1.14,1.43)$ & $<0.01$ & $1.21(1.07,1.36)$ & $<0.01$ \\
\hline$\geq 28.1$ & $2.24(2.02,2.49)$ & $<0.01$ & $2.10(1.89,2.34)$ & $<0.01$ & $1.47(1.31,1.66)$ & $<0.01$ \\
\hline$P$ trend & $<0.01$ & & $<0.01$ & & $<0.01$ & \\
\hline \multicolumn{7}{|l|}{ Quintiles } \\
\hline$<19.6$ & 1.0 (ref) & & 1.0 (ref) & & 1.0 (ref) & \\
\hline$\geq 19.6,<23.2$ & $1.09(0.93,1.27)$ & 0.27 & $1.00(0.85,1.16)$ & 0.96 & $1.04(0.89,1.23)$ & 0.62 \\
\hline$\geq 23.2,<26.7$ & $1.31(1.13,1.52)$ & $<0.01$ & $1.19(1.02,1.38)$ & 0.02 & $1.16(0.99,1.35)$ & 0.06 \\
\hline$\geq 26.7,<31.8$ & $1.84(1.60,2.12)$ & $<0.01$ & $1.63(1.41,1.88)$ & $<0.01$ & $1.39(1.20,1.61)$ & $<0.01$ \\
\hline$\geq 31.8$ & $2.50(2.18,2.86)$ & $<0.01$ & $2.31(2.01,2.65)$ & $<0.01$ & $1.50(1.30,1.74)$ & $<0.01$ \\
\hline$P$ trend & $<0.01$ & & $<0.01$ & & $<0.01$ & \\
\hline \multicolumn{7}{|c|}{ 365-day all-cause mortality } \\
\hline \multicolumn{7}{|c|}{ Tertiles } \\
\hline$<22.1$ & 1.0 (ref) & & 1.0 (ref) & & 1.0 (ref) & \\
\hline$\geq 22.1,<28.1$ & $1.41(1.28,1.56)$ & $<0.01$ & $1.31(1.18,1.44)$ & $<0.01$ & $1.22(1.10,1.35)$ & $<0.01$ \\
\hline$\geq 28.1$ & $2.18(1.99,2.39)$ & $<0.01$ & $2.06(1.88,2.27)$ & $<0.01$ & $1.46(1.32,1.62)$ & $<0.01$ \\
\hline$P$ trend & $<0.01$ & & $<0.01$ & & $<0.01$ & \\
\hline \multicolumn{7}{|l|}{ Quintiles } \\
\hline$<19.6$ & 1.0 (ref) & & 1.0 (ref) & & 1.0 (ref) & \\
\hline$\geq 19.6,<23.2$ & $1.11(0.97,1.27)$ & 0.12 & $1.01(0.88,1.16)$ & 0.87 & $1.06(0.92,1.22)$ & 0.41 \\
\hline$\geq 23.2,<26.7$ & $1.34(1.18,1.52)$ & $<0.01$ & $1.20(1.06,1.37)$ & $<0.01$ & $1.17(1.02,1.33)$ & 0.03 \\
\hline$\geq 26.7,<31.8$ & $1.83(1.62,2.07)$ & $<0.01$ & $1.61(1.42,1.82)$ & $<0.01$ & $1.36(1.20,1.55)$ & $<0.01$ \\
\hline$\geq 31.8$ & $2.40(2.13,2.70)$ & $<0.01$ & $2.25(2.00,2.54)$ & $<0.01$ & $1.49(1.31,1.70)$ & $<0.01$ \\
\hline$P$ trend & $<0.01$ & & $<0.01$ & & $<0.01$ & \\
\hline
\end{tabular}

HR: hazard ratio; CI: confidence interval. Models were derived from Cox proportional hazards regression models. Nonadjusted model, adjusted for none. Adjust I model, adjusted for age, ethnicity, and gender. Adjust II model, adjusted for age, ethnicity, gender, acute kidney injury stage, congestive heart failure, atrial fibrillation, liver disease, coronary artery disease, stroke, malignancy, respiratory failure, pneumonia, sodium, potassium, chloride, BUN, INR, APTT, platelet, WBC, hematocrit, creatinine, glucose, bicarbonate, vasopressor use, heart rate, systolic blood pressure, diastolic blood pressure, respiration rate, temperature, $\mathrm{SPO}_{2}$, SOFA, SAPSII, and renal replacement therapy.

neutrophils percentage, albumin, and SOFA score. Moreover, the area under the curve (AUC) was calculated to evaluate the quality of NPAR as a predictor of 30-day all-cause mortality. All statistical analyses were performed using EmpowerStats version 2.17 .8 (http://www.empowerstats.com/cn/, X\&Y solutions, Inc., Boston, $\mathrm{MA})$ and $R$ software version 3.42; $P<0.05$ was considered statistically significant.

\section{Results}

3.1. Subject Characteristics. A total of 7,481 eligible subjects were enrolled (Figure 1). Characteristics of the study patients stratified by NPARs tertiles are displayed in Table 1. A total of 2,492 patients were in the low-NPAR group (tertile 1: NPAR <22.1), 2,494 patients were in the mid-NPAR group (tertile 2: 22.1-28.1), and 2,495 patients were in the highNPAR group (tertile 3 : NPAR $\geq 28.1$ ). The subjects included 3,245 women and 4,236 men, most of whom were white. Patients with high NPAR values (NPAR $\geq 28.1$ ) were more likely to receive renal replacement therapy and vasopressors and to report a history of stroke, liver disease, malignancy, and respiratory failure; they also had lower SBP, DBP, MBP, bicarbonate, hematocrit, and hemoglobin; finally, they also had higher levels of heart rate, respiratory rate, creatinine, chloride, platelet, BUN, WBC, PT, APTT, SOFA, SAPSI, ICU LOS, and mortality. 
3.2. NPAR as a Predictor of the Clinical Endpoints. In model I, after adjustments for age, ethnicity, and gender, higher NPARs were associated with increased risk of all-cause mortality than were the first tertile $(<22.1)$ or quintile $(<19.6)$. In model II, after adjusting for more confounding factors, NPAR was also an independent predictor of 30-, 90-, and 365-day all-cause mortality in critically ill patients with AKI (tertile 3 versus tertile 1: adjusted HR, 95\% CI: 1.48, $1.30-1.69 ; 1.47,1.31-1.66 ; 1.46,1.32-1.62, P$ trend $<0.01)$. A similar trend was observed in NPAR group inclusion according to quintiles (Table 2). Moreover, the ROC curves were generated, and we found that the AUCs for NPAR, neutrophils percentage, albumin, and SOFA score were $0.693,0.538,0.633$, and 0.758 , respectively (Figure 2). Comparing AUCs, NPAR was found to be lower than SOFA score but was a better predictor than neutrophil percentage or albumin alone $(P<0.01)$.

3.3. Subgroup Analyses. There were no significant interactions in most strata in the subgroup analyses (Table 3 ). Patients with high values of potassium, platelet, hematocrit, bicarbonate, and SBP had higher risks of all-cause mortality for high NPAR. Similarly, patients with SOFA scores $<4$, SAPSII scores $<34$, temperature $<36.5^{\circ} \mathrm{C}$, heart rate $<80$ beats/minute, and glucose $<119.9 \mathrm{mg} / \mathrm{dl}$ were at increased risk with a NPAR $\geq 28.1$.

\section{Discussion}

We demonstrated that higher NPARs were associated with an increased risk of 30-, 90-, and 365-day all-cause mortality in critically ill patients with AKI after adjustments for age, ethnicity, and gender. Furthermore, after adjusting for more confounding factors, NPAR was also an independent predictor of all-cause mortality in these patients. Moreover, NPAR was found to be a better predictor than neutrophil percentage or albumin alone. There were no significant interactions in most strata in the subgroup analyses. To our knowledge, our study is the first to find that increased NPAR was independently associated with poor prognosis in critically ill patients with AKI.

Several studies have shown that AKI was associated with local and systemic inflammatory responses [21, 22]; therefore, as markers of inflammation and immune responses, neutrophil and albumin have been shown to provide additional information regarding the prognosis of AKI $[23,24]$. Combinations generating new biomarkers, including neutrophil-to-lymphocyte (NLR) and plateletto-lymphocyte ratio (PLR), are good prognostic indicators in patients with AKI $[25,26]$. Several studies have shown that hypoalbuminemia is a risk factor for the development and poor prognosis of AKI in critical illness [27-29]. Serum albumin protects the kidneys from toxic substances and maintains optimal colloid pressure to ensure renal perfusion [30]. Tawfik et al. [12] and Tingle et al. [13] suggested that NAR is an independent prognostic marker for survival in patients with solid tumors. The findings of the present study suggested that NPAR was an

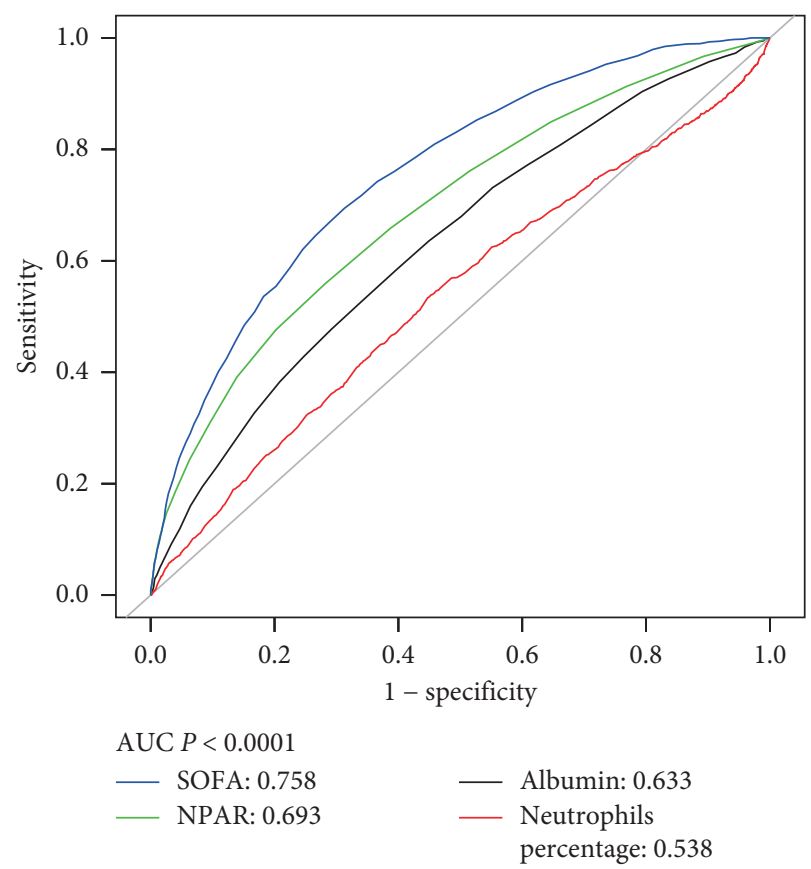

FIGURE 2: ROC curves for the prediction of 30-day all-cause mortality in critically ill patients with AKI. AUCs for NPAR, neutrophils percentage, albumin, and SOFA score were 0.693, $0.538,0.633$, and 0.758 .

independent predictor of all-cause mortality in critically ill patients with AKI and was a better predictor than neutrophil percentage or albumin alone. Therefore, we have reason to believe that NPAR has important clinical significance.

AKI involves a complex physiological process caused by a series of factors, and its pathogenesis remains unclear [31]. Previous studies have proposed several possible explanations, one of which is that high circulating levels of inflammatory mediators are crucial causes of AKI. The known inflammatory mediators associated with AKI and its prognosis include neutrophils, lymphocytes, platelets, interleukin- (IL-) 6, IL-10, tumor necrosis factor receptor(TNF-R-) I, TNF-R-II, C-reactive protein (CRP), albumin concentrations, and red blood cell distribution width (RDW) [32-34]. Another possible pathogenesis of AKI is impaired renal blood flow autoregulation [35]. Decreased renal blood flow depletes intracellular ATP, destroys intracellular calcium homeostasis, generates free radicals, activates inflammatory pathways, and destroys the integrity of the cytoskeleton $[36,37]$. These lesions eventually lead to hypoxic damage to tubular cells, and damaged cells form casts obstruct renal tubules.

There were some limitations in our study. First, the study had a single-center retrospective design, and biases were inevitable. Second, we calculated NPAR only upon admission to the ICU; a single measure of NPAR may affect the accuracy of the results. Third, although we did our best to use a multivariate model to control bias, there remain numerous other known and unknown factors. Finally, retrospective of databases has many defects; therefore, multicenter, prospective studies are needed to confirm these findings. 
TABLE 3: Subgroup analysis of the associations between the neutrophil percentage-to-albumin ratios and 30-day all-cause mortality.

\begin{tabular}{|c|c|c|c|c|c|}
\hline & $N$ & & phil percentage-to & n ratios & $P$ for interaction \\
\hline & $N$ & $<22.1$ & $\geq 22.1,<28.1$ & $\geq 28.1$ & $P$ Ior interaction \\
\hline $\mathrm{CHF}$ & & & & & 0.92 \\
\hline No & 6213 & 1.0 (ref) & $1.28(1.10,1.48)$ & $2.06(1.81,2.35)$ & \\
\hline Yes & 1268 & 1.0 (ref) & $1.31(0.93,1.84)$ & $2.25(1.61,3.14)$ & \\
\hline AFIB & & & & & 0.22 \\
\hline No & 5505 & 1.0 (ref) & $1.33(1.13,1.56)$ & $2.22(1.92,2.58)$ & \\
\hline Yes & 1976 & 1.0 (ref) & $1.08(0.86,1.36)$ & $1.80(1.45,2.23)$ & \\
\hline $\mathrm{CAD}$ & & & & & 0.06 \\
\hline No & 5853 & 1.0 (ref) & $1.15(0.99,1.34)$ & $1.90(1.66,2.18)$ & \\
\hline Yes & 1628 & 1.0 (ref) & $1.70(1.27,2.27)$ & $2.67(2.00,3.55)$ & \\
\hline Stroke & & & & & 0.22 \\
\hline No & 6856 & 1.0 (ref) & $1.29(1.11,1.49)$ & $2.23(1.95,2.55)$ & \\
\hline Yes & 625 & 1.0 (ref) & $1.23(0.88,1.71)$ & $1.65(1.18,2.32)$ & \\
\hline Malignancy & & & & & 0.03 \\
\hline No & 6073 & 1.0 (ref) & $1.37(1.17,1.59)$ & $2.21(1.91,2.55)$ & \\
\hline Yes & 1408 & 1.0 (ref) & $1.01(0.77,1.31)$ & $1.61(1.29,2.02)$ & \\
\hline Liver disease & & & & & 0.32 \\
\hline No & 6680 & 1.0 (ref) & $1.19(1.03,1.38)$ & $2.01(1.76,2.30)$ & \\
\hline Yes & 801 & 1.0 (ref) & $1.39(0.98,1.97)$ & $1.86(1.35,2.57)$ & \\
\hline Respiratory failure & & & & & $<0.01$ \\
\hline No & 4302 & 1.0 (ref) & $1.33(1.09,1.64)$ & $2.48(2.05,3.01)$ & \\
\hline Yes & 3179 & 1.0 (ref) & $1.10(0.92,1.31)$ & $1.49(1.27,1.75)$ & \\
\hline Pneumonia & & & & & 0.05 \\
\hline No & 5031 & 1.0 (ref) & $1.38(1.16,1.64)$ & $2.27(1.94,2.66)$ & \\
\hline Yes & 2450 & 1.0 (ref) & $1.04(0.84,1.28)$ & $1.71(1.41,2.07)$ & \\
\hline AKI stage & & & & & 0.02 \\
\hline Stage 1 & 1682 & 1.0 (ref) & $1.11(0.83,1.48)$ & $2.36(1.82,3.08)$ & \\
\hline Stage 2 & 1235 & 1.0 (ref) & $1.08(0.77,1.51)$ & $1.44(1.05,1.98)$ & \\
\hline Stage 3 & 4564 & $1.0(\mathrm{ref})$ & $1.35(1.14,1.59)$ & $2.16(1.85,2.52)$ & \\
\hline WBC (109/L) & & & & & 0.07 \\
\hline$<7.6$ & 2449 & 1.0 (ref) & $1.19(0.95,1.49)$ & $1.85(1.50,2.29)$ & \\
\hline$\geq 7.6,<12.1$ & 2528 & 1.0 (ref) & $1.58(1.23,2.03)$ & $2.53(1.98,3.22)$ & \\
\hline$\geq 12.1$ & 2502 & 1.0 (ref) & $0.98(0.78,1.23)$ & $1.61(1.31,1.99)$ & \\
\hline Sodium $(\mathrm{mmol} / \mathrm{L})$ & & & & & 0.09 \\
\hline$<134$ & 2010 & 1.0 (ref) & $1.05(0.82,1.35)$ & $1.85(1.48,2.30)$ & \\
\hline$\geq 134,<138$ & 2333 & 1.0 (ref) & $1.40(1.11,1.76)$ & $1.87(1.49,2.34)$ & \\
\hline$\geq 138$ & 3136 & 1.0 (ref) & $1.27(1.02,1.57)$ & $2.34(1.92,2.85)$ & \\
\hline BUN (mg/dl) & & & & & 0.28 \\
\hline$<17$ & 2295 & 1.0 (ref) & $1.45(1.09,1.94)$ & $1.90(1.44,2.52)$ & \\
\hline$\geq 17,<34$ & 2677 & 1.0 (ref) & $1.19(0.96,1.48)$ & $2.06(1.68,2.52)$ & \\
\hline$\geq 34$ & 2506 & 1.0 (ref) & $1.02(0.83,1.26)$ & $1.68(1.39,2.03)$ & \\
\hline INR & & & & & 0.06 \\
\hline$<1.2$ & 2404 & 1.0 (ref) & $1.36(1.05,1.76)$ & $2.52(1.96,3.24)$ & \\
\hline$\geq 1.2,<1.4$ & 2176 & 1.0 (ref) & $1.06(0.81,1.39)$ & $1.59(1.24,2.05)$ & \\
\hline$\geq 1.4$ & 2639 & 1.0 (ref) & $1.11(0.91,1.35)$ & $1.62(1.36,1.93)$ & \\
\hline Potassium $(\mathrm{mmol} / \mathrm{L})$ & & & & & $<0.01$ \\
\hline$<3.5$ & 2415 & 1.0 (ref) & $1.06(0.83,1.34)$ & $1.52(1.23,1.88)$ & \\
\hline$\geq 3.5,<4$ & 2560 & 1.0 (ref) & $1.23(0.97,1.56)$ & $2.24(1.80,2.79)$ & \\
\hline$\geq 4$ & 2505 & 1.0 (ref) & $1.40(1.12,1.75)$ & $2.49(2.02,3.06)$ & \\
\hline APTT (seconds) & & & & & 0.48 \\
\hline$<26.7$ & 2402 & 1.0 (ref) & $1.29(1.00,1.67)$ & $2.11(1.63,2.72)$ & \\
\hline$\geq 26.7,<32.2$ & 2404 & 1.0 (ref) & $1.29(1.01,1.66)$ & $1.91(1.52,2.41)$ & \\
\hline$\geq 32.2$ & 2405 & 1.0 (ref) & $1.05(0.85,1.28)$ & $1.65(1.37,1.98)$ & \\
\hline Platelet $(109 / \mathrm{L})$ & & & & & 0.01 \\
\hline$<139$ & 2468 & 1.0 (ref) & $1.10(0.90,1.35)$ & $1.61(1.35,1.93)$ & \\
\hline$\geq 139,<223$ & 2492 & 1.0 (ref) & $1.56(1.23,1.99)$ & $2.39(1.89,3.03)$ & \\
\hline$\geq 223$ & 2518 & 1.0 (ref) & $1.30(1.00,1.71)$ & $2.47(1.93,3.15)$ & \\
\hline Hematocrit (\%) & & & & & $<0.01$ \\
\hline$<26.9$ & 2470 & 1.0 (ref) & $0.88(0.69,1.12)$ & $1.60(1.31,1.95)$ & \\
\hline$\geq 26.9,<32.3$ & 2515 & 1.0 (ref) & $1.29(1.02,1.63)$ & $1.85(1.48,2.31)$ & \\
\hline
\end{tabular}


TABLE 3: Continued.

\begin{tabular}{|c|c|c|c|c|c|}
\hline & $N$ & & phil percentage-to & n ratios & $P$ for interaction \\
\hline & $N$ & $<22.1$ & $\geq 22.1,<28.1$ & $\geq 28.1$ & $P$ lor interaction \\
\hline$\geq 32.3$ & 2495 & 1.0 (ref) & $1.57(1.26,1.96)$ & $3.05(2.44,3.81)$ & \\
\hline $\begin{array}{l}\text { Creatinine }(\mathrm{mEq} / \mathrm{L}) \\
\quad<0.9 \\
\geq 0.9,<1.5 \\
\geq 1.5\end{array}$ & $\begin{array}{l}2440 \\
2490 \\
2549 \\
\end{array}$ & $\begin{array}{l}1.0 \text { (ref) } \\
1.0 \text { (ref) } \\
1.0(\text { ref }) \\
\end{array}$ & $\begin{array}{l}1.59(1.22,2.09) \\
1.21(0.96,1.52) \\
1.00(0.82,1.22) \\
\end{array}$ & $\begin{array}{l}2.41(1.88,3.10) \\
2.03(1.62,2.53) \\
1.67(1.39,2.00) \\
\end{array}$ & 0.13 \\
\hline $\begin{array}{l}\text { Bicarbonate }(\mathrm{mg} / \mathrm{dl}) \\
\quad<18 \\
\geq 18,<23 \\
\geq 23\end{array}$ & $\begin{array}{l}2068 \\
2775 \\
2636 \\
\end{array}$ & $\begin{array}{l}1.0 \text { (ref) } \\
1.0 \text { (ref) } \\
1.0 \text { (ref) }\end{array}$ & $\begin{array}{l}1.00(0.81,1.24) \\
1.24(0.99,1.57) \\
1.51(1.18,1.94) \\
\end{array}$ & $\begin{array}{l}1.38(1.14,1.68) \\
2.08(1.68,2.58) \\
2.72(2.15,3.45) \\
\end{array}$ & $<0.01$ \\
\hline $\begin{array}{l}\text { Glucose }(\mathrm{mg} / \mathrm{dl}) \\
\quad<119.9 \\
\geq 119.9,<152.8 \\
\geq 152.8\end{array}$ & $\begin{array}{l}2485 \\
2484 \\
2485 \\
\end{array}$ & $\begin{array}{l}1.0(\text { ref }) \\
1.0(\text { ref }) \\
1.0(\text { ref })\end{array}$ & $\begin{array}{l}1.43(1.11,1.84) \\
1.39(1.10,1.76) \\
1.00(0.82,1.24) \\
\end{array}$ & $\begin{array}{l}2.63(2.10,3.30) \\
2.16(1.73,2.71) \\
1.62(1.33,1.96) \\
\end{array}$ & 0.03 \\
\hline $\begin{array}{l}\text { Chloride }(\mathrm{mmol} / \mathrm{L}) \\
\quad<99 \\
\geq 99,<104 \\
\geq 104 \\
\end{array}$ & $\begin{array}{l}2206 \\
2370 \\
2903 \\
\end{array}$ & $\begin{array}{l}1.0(\mathrm{ref}) \\
1.0(\mathrm{ref}) \\
1.0(\mathrm{ref})\end{array}$ & $\begin{array}{l}1.17(0.94,1.47) \\
1.35(1.07,1.70) \\
1.24(0.98,1.58) \\
\end{array}$ & $\begin{array}{l}1.90(1.53,2.35) \\
2.33(1.87,2.89) \\
2.12(1.72,2.63) \\
\end{array}$ & 0.76 \\
\hline $\begin{array}{l}\text { SBP }(\mathrm{mmHg}) \\
\quad<108 \\
\quad \geq 108,<123 \\
\geq 123\end{array}$ & $\begin{array}{l}2486 \\
2485 \\
2486\end{array}$ & $\begin{array}{l}1.0 \text { (ref) } \\
1.0 \text { (ref) } \\
1.0 \text { (ref) }\end{array}$ & $\begin{array}{l}0.99(0.81,1.21) \\
1.47(1.15,1.88) \\
1.40(1.08,1.81) \\
\end{array}$ & $\begin{array}{l}1.53(1.28,1.83) \\
2.21(1.75,2.79) \\
2.40(1.87,3.07)\end{array}$ & 0.02 \\
\hline $\begin{array}{l}\text { DBP }(\mathrm{mmHg}) \\
\quad<55 \\
\quad \geq 55,<64 \\
\geq 64 \\
\end{array}$ & $\begin{array}{l}2485 \\
2486 \\
2486 \\
\end{array}$ & $\begin{array}{l}1.0(\text { ref }) \\
1.0(\text { ref }) \\
1.0(\text { ref })\end{array}$ & $\begin{array}{l}1.19(0.96,1.48) \\
1.07(0.85,1.35) \\
1.50(1.18,1.92) \\
\end{array}$ & $\begin{array}{l}2.01(1.65,2.44) \\
1.81(1.47,2.23) \\
2.22(1.74,2.83) \\
\end{array}$ & 0.36 \\
\hline $\begin{array}{l}\text { Heart rate (beats/minute) } \\
\quad<80 \\
\geq 80,<96 \\
\geq 96\end{array}$ & $\begin{array}{l}2487 \\
2488 \\
2490 \\
\end{array}$ & $\begin{array}{l}1.0 \text { (ref) } \\
1.0 \text { (ref) } \\
1.0(\text { ref })\end{array}$ & $\begin{array}{l}1.52(1.20,1.92) \\
1.26(0.99,1.61) \\
1.00(0.80,1.24) \\
\end{array}$ & $\begin{array}{l}2.63(2.10,3.31) \\
2.04(1.63,2.55) \\
1.54(1.27,1.86) \\
\end{array}$ & 0.02 \\
\hline $\begin{array}{l}\text { Respiratory rate (beats/minute) } \\
\quad<18 \\
\quad \geq 18,<22 \\
\geq 22\end{array}$ & $\begin{array}{l}2483 \\
2482 \\
2485 \\
\end{array}$ & $\begin{array}{l}1.0(\mathrm{ref}) \\
1.0(\mathrm{ref}) \\
1.0(\mathrm{ref}) \\
\end{array}$ & $\begin{array}{l}1.72(1.31,2.25) \\
1.28(1.01,1.64) \\
0.93(0.76,1.13) \\
\end{array}$ & $\begin{array}{l}2.62(2.03,3.38) \\
2.45(1.96,3.06) \\
1.40(1.17,1.67) \\
\end{array}$ & $<0.01$ \\
\hline $\begin{array}{l}\text { Temperature }\left({ }^{\circ} \mathrm{C}\right) \\
\quad<36.5 \\
\geq 36.5,<37.1 \\
\geq 37.1\end{array}$ & $\begin{array}{l}2464 \\
2465 \\
2470 \\
\end{array}$ & $\begin{array}{l}1.0(\text { ref }) \\
1.0(\text { ref }) \\
1.0(\text { ref })\end{array}$ & $\begin{array}{l}1.47(1.19,1.83) \\
1.28(1.00,1.66) \\
1.04(0.83,1.32) \\
\end{array}$ & $\begin{array}{l}2.37(1.94,2.88) \\
2.37(1.87,2.99) \\
1.55(1.25,1.93) \\
\end{array}$ & 0.04 \\
\hline $\begin{array}{l}\text { SPO2 }(\%) \\
\quad<96.5 \\
\quad \geq 96.5,<98.3 \\
\geq 98.3 \\
\end{array}$ & $\begin{array}{l}2478 \\
2491 \\
2485 \\
\end{array}$ & $\begin{array}{l}1.0(\text { ref }) \\
1.0(\text { ref }) \\
1.0(\text { ref })\end{array}$ & $\begin{array}{l}1.06(0.86,1.30) \\
1.17(0.91,1.51) \\
1.61(1.26,2.05) \\
\end{array}$ & $\begin{array}{l}2.01(1.67,2.43) \\
2.05(1.63,2.57) \\
2.28(1.81,2.87) \\
\end{array}$ & 0.07 \\
\hline $\begin{array}{l}\text { SOFA score } \\
\quad<4 \\
\quad \geq 4,<7 \\
\geq 7\end{array}$ & $\begin{array}{l}2295 \\
2581 \\
2605 \\
\end{array}$ & $\begin{array}{l}1.0(\mathrm{ref}) \\
1.0(\mathrm{ref}) \\
1.0(\mathrm{ref})\end{array}$ & $\begin{array}{l}1.49(1.09,2.05) \\
1.24(0.96,1.59) \\
1.02(0.85,1.22) \\
\end{array}$ & $\begin{array}{l}2.52(1.83,3.45) \\
2.01(1.59,2.54) \\
1.34(1.14,1.58) \\
\end{array}$ & $<0.01$ \\
\hline $\begin{array}{l}\text { SAPSII score } \\
\quad<34 \\
\quad \geq 34,<47 \\
\geq 47\end{array}$ & $\begin{array}{l}2375 \\
2607 \\
2499 \\
\end{array}$ & $\begin{array}{l}1.0(\text { ref }) \\
1.0(\text { ref }) \\
1.0(\text { ref }) \\
\end{array}$ & $\begin{array}{l}1.42(0.93,2.16) \\
1.36(1.06,1.74) \\
1.02(0.86,1.21) \\
\end{array}$ & $\begin{array}{l}2.93(1.97,4.36) \\
1.98(1.56,2.52) \\
1.27(1.09,1.48) \\
\end{array}$ & $<0.01$ \\
\hline $\begin{array}{l}\text { Vasopressor use } \\
\text { No } \\
\text { Yes } \\
\end{array}$ & $\begin{array}{l}4472 \\
3009 \\
\end{array}$ & $\begin{array}{l}1.0 \text { (ref) } \\
1.0 \text { (ref) }\end{array}$ & $\begin{array}{l}1.48(1.20,1.83) \\
1.03(0.86,1.22)\end{array}$ & $\begin{array}{l}2.66(2.18,3.25) \\
1.40(1.20,1.64)\end{array}$ & $<0.01$ \\
\hline $\begin{array}{l}\text { Renal replacement therapy } \\
\text { No } \\
\text { Yes }\end{array}$ & $\begin{array}{c}6692 \\
789 \\
\end{array}$ & $\begin{array}{l}1.0 \text { (ref) } \\
1.0 \text { (ref) }\end{array}$ & $\begin{array}{l}1.24(1.07,1.43) \\
1.16(0.83,1.63)\end{array}$ & $\begin{array}{l}2.17(1.90,2.48) \\
1.34(0.97,1.84)\end{array}$ & $<0.01$ \\
\hline
\end{tabular}

CHF: congestive heart failure; AFIB: atrial fibrillation; CAD: coronary artery disease; AKI: acute kidney injury; WBC: white blood cell; BUN: blood urea nitrogen; INR: international normalized ratio; APTT: activated partial thromboplastin time; SBP: systolic blood pressure; DBP: diastolic blood pressure; SOFA: sequential organ failure assessment; SAPSII: simplified acute physiology score II. 


\section{Conclusions}

We demonstrated that higher NPARs were associated with increased risk of 30-, 90-, and 365-day all-cause mortality in critically ill patients with AKI. Nevertheless, these findings need to be confirmed by large prospective multicenter studies.

\section{Data Availability}

The clinical data used to support the findings of this study were supplied by Monitoring in Intensive Care Database III version 1.4 (MIMIC-III v.1.4). Although the database is publicly and freely available, researchers must complete the National Institutes of Health's web-based course known as Protecting Human Research Participants to apply for permission to access the database.

\section{Ethical Approval}

The MIMIC-III database has received ethical approval from the institutional review boards (IRBs) at Beth Israel Deaconess Medical Center and Massachusetts Institute of Technology. Because the database does not contain protected health information, a waiver of the requirement for informed consent was included in the IRB approval.

\section{Disclosure}

The funders of the project were not involved in study design, collection, data analysis, writing of the report, and publication.

\section{Conflicts of Interest}

The authors declare that they have no conflicts of interest.

\section{Acknowledgments}

This research was supported by the Zhejiang Provincial Natural Science Foundation of China (Grants nos. LY19H150002 and LY13H150007), the Scientific Research Foundation of Wenzhou (Grants nos. Y20180515 and Y20150038), and the Clinical Research Foundation of the 2nd Affiliated Hospital of Wenzhou Medical University (SAHoWMU-CR2019-11-423).

\section{Supplementary Materials}

The occurrence of AKI was determined on the basis of Kidney Disease: Improving Global Outcomes (KDIGO) definition [17], and Structured Query Language (SQL) for extracting AKI was included in supplementary material. (Supplementary Materials)

\section{References}

[1] R. Bellomo, J. A. Kellum, and C. Ronco, "Acute kidney injury,” The Lancet, vol. 380, no. 9843, pp. 756-766, 2012.
[2] S. Uchino, J. A. Kellum, R. Bellomo et al., "Acute renal failure in critically ill patients," Jama, vol. 294, no. 7, pp. 813-818, 2005.

[3] A. Khwaja, "KDIGO clinical practice guidelines for acute kidney injury," Nephron Clinical Practice, vol. 120, no. 4, pp. c179-c184, 2012.

[4] J. H. Westhoff, F. S. Seibert, S. Waldherr et al., "Urinary calprotectin, kidney injury molecule-1, and neutrophil gelatinase-associated lipocalin for the prediction of adverse outcome in pediatric acute kidney injury," European Journal of Pediatrics, vol. 176, no. 6, pp. 745-755, 2017.

[5] W. R. Zhang and C. R. Parikh, "Biomarkers of acute and chronic kidney disease," Annual Review of Physiology, vol. 81, pp. 309-333, 2019.

[6] I. Mami, Q. Tavernier, N. Bouvier et al., "A novel extrinsic pathway for the unfolded protein response in the kidney," Journal of the American Society of Nephrology, vol. 27, no. 9, pp. 2670-2683, 2016.

[7] H. Rabb, M. D. Griffin, D. B. McKay et al., "Inflammation in AKI: current understanding, key questions, and knowledge gaps," Journal of the American Society of Nephrology, vol. 27, no. 2, pp. 371-379, 2016.

[8] M. P. Jansen, D. Emal, G. J. Teske, M. C. Dessing, S. Florquin, and J. J. Roelofs, "Release of extracellular DNA influences renal ischemia reperfusion injury by platelet activation and formation of neutrophil extracellular traps," Kidney International, vol. 91, no. 2, pp. 352-364, 2017.

[9] S. A. Namendys-Silva, M. O. Gonzalez-Herrera, J. TexcocanoBecerra, and A. Herrera-Gomez, "Hypoalbuminemia in critically ill patients with cancer: incidence and mortality," The American Journal of Hospice \& Palliative Care, vol. 28, no. 4, pp. 253-257, 2011.

[10] M. Y. Yu, S. W. Lee, S. H. Baek et al., "Hypoalbuminemia at admission predicts the development of acute kidney injury in hospitalized patients: a retrospective cohort study," PloS One, vol. 12, no. 7, Article ID e0180750, 2017.

[11] S. N. Murat, A. Kurtul, and M. Yarlioglues, "Impact of serum albumin levels on contrast-induced acute kidney injury in patients with acute coronary syndromes treated with percutaneous coronary intervention," Angiology, vol. 66, no. 8, pp. 732-737, 2015.

[12] B. Tawfik, A. A. Mokdad, P. M. Patel, H. C. Li, and S. Huerta, "The neutrophil to albumin ratio as a predictor of pathological complete response in rectal cancer patients following neoadjuvant chemoradiation," Anti-Cancer Drugs, vol. 27, no. 9, pp. 879-883, 2016.

[13] S. J. Tingle, G. R. Severs, M. Goodfellow, J. A. Moir, and S. A. White, "NARCA: a novel prognostic scoring system using neutrophil-albumin ratio and Ca19-9 to predict overall survival in palliative pancreatic cancer," Journal of Surgical Oncology, vol. 118, no. 4, pp. 680-686, 2018.

[14] B. Wang, D. Li, Y. Gong, B. Ying, and B. Cheng, "Association of serum total and ionized calcium with all-cause mortality incritically ill patients with acute kidney injury," Clinica Chimica Acta, vol. 494, pp. 94-99, 2019.

[15] B. Wang, Y. Gong, B. Ying, and B. Cheng, "Relation between red cell distribution width and mortality in critically ill patients with acute respiratory distress syndrome," BioMed Research International, vol. 2019, p. 1942078, 2019.

[16] A. E. Johnson, T. J. Pollard, L. Shen et al., "MIMIC-III, a freely accessible critical care database," Scientific Data, vol. 3, p. 160035, 2016.

[17] R. L. Mehta, J. A. Kellum, S. V. Shah et al., “Acute Kidney Injury N: acute Kidney Injury Network: report of an initiative 
to improve outcomes in acute kidney injury," Critical Care, vol. 11, no. 2, p. R31, 2007.

[18] J. L. Vincent, R. Moreno, J. Takala et al., "The SOFA (Sepsisrelated organ failure assessment) score to describe organ dysfunction/failure. On behalf of the working group on sepsis-related problems of the European society of intensive care medicine," Intensive Care Medicine, vol. 22, no. 7, pp. 707-710, 1996.

[19] J. R. Le Gall, S. Lemeshow, and F. Saulnier, "A new simplified acute physiology score (SAPS II) based on a European/North American multicenter study," Jama, vol. 270, no. 24, pp. 2957-2963, 1993.

[20] G. Maldonado and S. Greenland, "Simulation study of confounder-selection strategies," American Journal of Epidemiology, vol. 138, no. 11, pp. 923-936, 1993.

[21] K. Doi and H. Rabb, "Impact of acute kidney injury on distant organ function: recent findings and potential therapeutic targets," Kidney International, vol. 89, no. 3, pp. 555-564, 2016.

[22] Y. Karabag, M. Cagdas, I. Rencuzogullari et al., "The C-reactive protein to albumin ratio predicts acute kidney injury in patients with ST-segment elevation myocardial infarction undergoing primary percutaneous coronary intervention," Heart, Lung \& Circulation, vol. 28, no. 11, pp. 1638-1645, 2018.

[23] B. Wang, H. Lu, Y. Gong, B. Ying, and B. Cheng, "The association between red blood cell distribution width and mortality in critically ill patients with acute kidney injury," BioMed Research International, vol. 2018, p. 9658216, 2018.

[24] A. Zarjou and A. Agarwal, "Sepsis and acute kidney injury," Journal of the American Society of Nephrology, vol. 22, no. 6, pp. 999-1006, 2011.

[25] X. Bu, L. Zhang, P. Chen, and X. Wu, "Relation of neutrophilto-lymphocyte ratio to acute kidney injury in patients with sepsis and septic shock: a retrospective study," International Immunopharmacology, vol. 70, pp. 372-377, 2019.

[26] C. F. Zheng, W. Y. Liu, F. F. Zeng et al., "Prognostic value of platelet-to-lymphocyte ratios among critically ill patients with acute kidney injury," Critical Care, vol. 21, no. 1, p. 238, 2017.

[27] C. J. Wiedermann, W. Wiedermann, and M. Joannidis, "Hypoalbuminemia and acute kidney injury: a meta-analysis of observational clinical studies," Intensive Care Medicine, vol. 36, no. 10, pp. 1657-1665, 2010.

[28] B. H. Sang, J. Y. Bang, J. G. Song, and G. S. Hwang, "Hypoalbuminemia within two postoperative days is an independent risk factor for acute kidney injury following living donor liver transplantation: a propensity score analysis of 998 consecutive patients," Critical Care Medicine, vol. 43, no. 12, pp. 2552-2561, 2015.

[29] C. Thongprayoon, W. Cheungpasitporn, M. A. Mao, A. Sakhuja, and K. Kashani, "U-shape association of serum albumin level and acute kidney injury risk in hospitalized patients," PloS One, vol. 13, no. 6, Article ID e0199153, 2018.

[30] A. M. Contreras, M. Ramirez, L. Cueva, S. Alvarez, R. de Loza, and G. Gamba, "Low serum albumin and the increased risk of amikacin nephrotoxicity," Revista de investigacion clinica; organo del Hospital de Enfermedades de la Nutricion, vol. 46, no. 1, pp. 37-43, 1994.

[31] A. A. Ejaz and R. Mohandas, "Are diuretics harmful in the management of acute kidney injury?" Current Opinion in Nephrology and Hypertension, vol. 23, no. 2, pp. 155-160, 2014.

[32] Z. Forhecz, T. Gombos, G. Borgulya, Z. Pozsonyi, Z. Prohaszka, and L. Janoskuti, "Red cell distribution width in heart failure: prediction of clinical events and relationship with markers of ineffective erythropoiesis, inflammation, renal function, and nutritional state," American Heart Journal, vol. 158, no. 4, pp. 659-666, 2009.

[33] D. Payen, A. C. Lukaszewicz, M. Legrand et al., "A multicentre study of acute kidney injury in severe sepsis and septic shock: association with inflammatory phenotype and HLA genotype," PloS One, vol. 7, no. 6, Article ID e35838, 2012.

[34] M. Araujo, S. Q. Doi, C. E. Palant, E. S. Nylen, and K. L. Becker, "Procalcitonin induced cytotoxicity and apoptosis in mesangial cells: implications for septic renal injury," Inflammation Research Official Journal of the European Histamine Research Society, vol. 62, no. 10, pp. 887-894, 2013.

[35] H. M. Kwon, Y. J. Moon, K. W. Jung et al., "Low mean arterial blood pressure is independently associated with postoperative acute kidney injury after living donor liver transplantation: a propensity score weighing analysis," Annals of Transplantation, vol. 23, pp. 236-245, 2018.

[36] P. Devarajan, "Update on mechanisms of ischemic acute kidney injury," Journal of the American Society of Nephrology, vol. 17, no. 6, pp. 1503-1520, 2006.

[37] J. V. Bonventre and L. Yang, "Cellular pathophysiology of ischemic acute kidney injury," The Journal of Clinical Investigation, vol. 121, no. 11, pp. 4210-4221, 2011. 